FOLIA

Amazónica

Revista del Instituto de Investigaciones

de la Amazonía Peruana

\title{
IMPACTO DE LA CONSTRUCCIÓN DE LA CARRETERA IQUITOS-SARAMIRIZA SOBRE LOS BOSQUES Y TURBERAS DEL RÍO TIGRE, LORETO, PERÚ
}

\author{
Eurídice N. HONORIO CORONADO ${ }^{1 *}$, Armando MERCADO TORRES ${ }^{2}$, \\ Dennis DEL CASTILLO TORRES ${ }^{1}$, Nállarett DÁVILA CARDOZO ${ }^{1}$, \\ Manuel MARTÍN BRAÑAS ${ }^{1}$, Sandra RÍOS TORRES ${ }^{2}$, Timothy R. BAKER ${ }^{3}$, \\ Mariana MONTOYA ${ }^{2}$
}

\footnotetext{
1 Instituto de Investigaciones de la Amazonía Peruana (IIAP), Av. Abelardo Quiñonez km 2.5, Loreto, Perú.

2 Wildlife Conservation Society Peru, Lima, Perú.

3 School of Geography, Universidad de Leeds, Leeds, UK.

* Correo electrónico: eurihc@yahoo.com
}

\section{RESUMEN}

El proyecto de infraestructura que busca conectar Iquitos con Saramiriza incluye la construcción de una nueva carretera asfaltada de ca. $350 \mathrm{~km}$ a lo largo del río Tigre en la región Loreto. Este proyecto es una amenaza para la conservación de una de las áreas más extensas de bosque tropical del planeta y de las turberas más extensas y profundas de la cuenca amazónica. En este estudio, enfocado en el segundo tramo Huambé-Marsella, usamos un escenario habitual sin carretera ("business as usual") y un escenario con carretera para mostrar cómo la construcción de la carretera conduciría a la pérdida substancial de la cobertura de bosques y aumentaría las emisiones de $\mathrm{CO}_{2}$. La pérdida actual de la cobertura de bosques en la zona de influencia de $20 \mathrm{~km}$ alrededor del tramo de carretera Huambé-Marsella, estimada al 2018, fue de 3,4 \%. Utilizando una red neuronal de perceptrones multicapas (MLP), estimamos una deforestación de 80 071,11 ha y emisiones de 35,55 $\mathrm{Mt} \mathrm{CO}_{2}$-eq para el periodo 2019-2064 en el escenario habitual, mientras el escenario con la carretera generaría la mayor pérdida de bosques (303 248,79 ha) y emisiones (135,56 $\mathrm{Mt} \mathrm{CO}_{2}$ eq). Evitar la construcción de la carretera a lo largo del río Tigre prevendría la pérdida de 83 462,58 ha de 
bosques y la emisión de 41,49 $\mathrm{Mt} \mathrm{CO}_{2}$-eq del 2019 al 2030. Por lo tanto, el estado peruano debe evaluar propuestas alternativas de transporte para la zona. Por ejemplo, servicios con una flota fluvial mejorada podrían ser utilizados para el transporte de carga y personas, mientras un servicio de aerotaxis podría también ayudar a atender las necesidades de los comunidades indígenas y pobladores ribereños. El cumplimiento de los compromisos internacionales para reducir la emisión de gases de efecto invernadero será potencialmente imposible si se construye la carretera, debido a que se estima que las emisiones nacionales aumentarían en 1,31 \% del 2019 al 2030. La inversión no realizada en la carretera podría subvencionar el uso de una flota fluvial moderna, el servicio de aerotaxis y la implementación de medidas de conservación y manejo sostenible de los bosques y turberas amazónicas del río Tigre.

PALABRAS CLAVE: aguaje, deforestación, emisiones de gases de efecto invernadero, aprovechamiento sostenible, turba.

\title{
IMPACT OF THE CONSTRUCTION OF THE SARAMIRIZA-IQUITOS ROAD ON FORESTS AND PETLANDS IN THE RIO TIGRE, LORETO, PERU
}

\begin{abstract}
New infrastructure that seeks to connect Iquitos with Saramiriza includes building a $\sim 350$-km paved road alongside the Tigre river in Loreto. This project is a threat to the conservation of one of the largest areas of tropical forest on the planet and the largest and deepest peatlands in the Amazon basin. In this study, focused on the second section of the proposed road (Huambé - Marsella), we used a "business-as-usual" scenario (BAU) and a "road" scenario to show that road construction would lead to substantial loss of forest cover and increase $\mathrm{CO}_{2}$ emissions. The current loss of forest cover in a buffer zone of $20 \mathrm{~km}$ around the Huambé-Marsella road, estimated up to 2018, was $3.4 \%$. Using a multi-layer perceptron (MLP) neural network, we estimated deforestation of 80071.11 ha and emissions of 35.55 $\mathrm{Mt} \mathrm{CO}_{2}$-eq for the period 2019-2064 in the BAU scenario, while the scenario with the construction of the road would generate greater loss of forests (303 $248.79 \mathrm{ha}$ ) and higher emissions (135.56 $\mathrm{Mt} \mathrm{CO}_{2}$-eq). Avoiding the construction of the road alongside the Tigre river would therefore prevent the loss of 83462.58 ha of forests and the emission of $41.49 \mathrm{Mt} \mathrm{CO}_{2}$-eq from 2019 to 2030. Therefore, the Peruvian government should assess alternative proposals for transportation in this area. For example, improved fluvial boat
\end{abstract}


services could be used for the transport of cargo and people, while an air taxi service could also help to meet the needs of indigenous communities and riverine settlers. Compliance with international commitments to reduce greenhouse gas emissions will potentially be impossible if the road is constructed as it is estimated that will increase national emissions by $1.31 \%$ from 2019 to 2030 . The funds saved from not constructing the road could subsidize the use of a modern river fleet, the air taxi service and the implementation of conservation strategies and sustainable management of the Amazonian forests and peatlands of the Tigre river.

KEYWORDS: aguaje, deforestation, greenhouse gas emissions, sustainable use, peat. 


\section{INTRODUCCIÓN}

Los bosques amazónicos peruanos son reconocidos por su importancia en la mitigación del cambio climático debido a su potencial en el almacenamiento del carbono en la biomasa y el suelo y la remoción de los gases de efecto invernadero de la atmósfera (Baker et al., 2019; Vicuña et al., 2019). Sin embargo, las carreteras son uno de los principales promotores de su afectación, al incrementar el acceso a los bosques que son tumbados y quemados para la expansión de áreas agrícolas y urbanas (Mäki et al., 2001; Hyman \& Barona, 2010; Sánchez Cuervo et al., 2020). En la región Loreto, el nuevo proyecto de infraestructura que busca conectar la "aislada" ciudad de Iquitos con las ciudades de la sierra y la costa peruana presenta $800 \mathrm{~km}$ de longitud y está dividida en cinco tramos (1) IquitosHuambé, sobre la carretera prexistente camino a Nauta km 85, (2) Huambé-Marsella, un centro logístico petrolero sobre el Río Tigre, y donde se propone construir una nueva carretera de $350 \mathrm{~km}$ de longitud, (3) Marsella-Nuevo Andoas, aprovechando un camino prexistente de servicio a pozos petroleros de ca. $100 \mathrm{~km}$ de longitud y (4) Nuevo Andoas-Saramiriza, donde se propone construir una nueva carretera de $275 \mathrm{~km}$ de longitud. Este proyecto tendrá un alto costo de inversión y mantenimiento, y se estima que generará un alto impacto socioambiental, con una pérdida adicional de 35961 hectáreas de bosque al 2030 en una zona de influencia de $20 \mathrm{~km}$, y nuevos conflictos sociales (Vilela et al., 2020). El acceso a los territorios de las comunidades amazónicas antes inaccesibles favorece que la pérdida de los valores, prácticas y conocimientos sobre el manejo y conservación de los recursos se acelere (Martín et al., 2019). Entender la magnitud del impacto de una nueva carretera es necesario para evaluar otras alternativas de transporte que mejoren la conexión con las ciudades amazónicas, así como actividades económicas viables que mantengan los bosques en pie y sus servicios ecosistémicos, permitiendo que la región Loreto sea resiliente ante el cambio climático (Blanco-Gutiérrez et al., 2019).

La pérdida de la cobertura de bosque en la región Loreto aumentó en las dos últimas décadas, perdiendo 96922 hectáreas en el periodo 2001-2006, 142522 hectáreas en el periodo 2007-2012 y 190838 hectáreas en el periodo 2013-2018 (MINAM, 2019a). La apertura de una nueva carretera aumentaría la tasa de deforestación y promovería otros problemas relacionados como el tráfico de tierras, el incremento de la tala ilegal, la minería ilegal, los cultivos ilegales, la degradación de ecosistemas frágiles, entre otros. Los impactos de la construcción de una carretera no son ajenos a la región Loreto. Se cuenta con un registro histórico de lo ocurrido en el área de influencia de la carretera Iquitos-Nauta, cuya construcción inició en los años 70s y se extendió durante casi 30 años (Mäki et al., 2001). La deforestación de los bosques fue ocasionada por un incremento en la ocupación poblacional, la extracción irracional de los recursos y la escasa aplicación y cumplimiento de políticas adecuadas de uso del territorio. Los ecosistemas frágiles, como los varillales de arena blanca, fueron explotados para la extracción de madera redonda y en muchos casos destruidos debido a la extracción del sustrato mineral (arena) para la industria de la construcción (Mäki et al., 2001).

Los bosques en Loreto cubren una extensión de más de 35 millones de hectáreas y representan el $51 \%$ de los bosques amazónicos del Perú (MINAM, 2019a). En particular, los bosques de colinas altas presentan alta densidad de carbono almacenado sobre el suelo a nivel nacional (Asner et al., 2014), mientras los ecosistemas de turberas acumulan materia orgánica parcialmente descompuesta (turba) debajo del suelo y tienen 
la más alta concentración de carbono en toda la cuenca amazónica (Lähteenoja et al., 2009, 2012; Draper et al., 2014). La perturbación antrópica de los bosques y turberas debido a la remoción de la cobertura vegetal y/o el drenaje del agua promueve la emisión de gases de efecto invernadero, debido a la quema de la biomasa y la descomposición de la materia orgánica del suelo y la turba, contribuyendo al calentamiento global (Baker et al., 2019). En Perú, se estima que la conversión de los bosques amazónicos a usos agrícolas generó la emisión de 104,3 $\mathrm{Mt} \mathrm{CO}_{2}$-eq, lo que corresponde al $45 \%$ de las emisiones nacionales de gases de efecto invernadero (MINAM, 2019b). En Indonesia, se estima que quedan solo $29 \%$ de las turberas naturales y su conversión en plantaciones de palma aceitera y acacia, para la producción de pulpa y papel, ha contribuido con el $38 \%$ de las emisiones nacionales de gases de efecto invernadero (Warren et al., 2017). Sin embargo, la región Loreto aún presenta extensas áreas de bosques y turberas, almacenando 3 685,1 Mt $C$ en la vegetación que se encuentra arriba del suelo (Asner et al., 2014) y 3140 Mt C en las turberas de la cuenca del Pastaza-Marañón que consideran la vegetación arriba y la turba debajo del suelo (Draper et al., 2014). Por lo tanto, evitar la conversión de los bosques y turberas en Loreto es importante para mantener el servicio ecosistémico de almacenamiento de carbono.

Las áreas naturales protegidas nacionales y regionales, así como los territorios de las comunidades indígenas y algunas comunidades ribereñas en Loreto albergan grandes extensiones de bosques conservados donde se desarrolla un conjunto de actividades de protección y uso sostenible de los recursos (Oliveira et al., 2007; Blackman et al., 2017). Es así, que los bosques de Loreto representan una de las áreas más extensas y persistente de bosque tropical del planeta (Watson etal., 2018). Contribuyen con el bienestar de las poblaciones indígenas y ribereñas de Loreto (Martín et al., 2019) y la humanidad a nivel global al proveer bienes y servicios como alimentos, materias primas, la captura y almacenamiento del carbono y la regulación del clima (Foley et al., 2007; Laurance et al., 2009). La construcción de una nueva carretera es una amenaza para la conservación de estos bosques y el bienestar de las comunidades amazónicas. Por lo tanto, el objetivo del presente estudio fue evaluar cómo la pérdida de bosques incrementaría las emisiones de $\mathrm{CO}_{2}$ a la atmósfera bajo dos escenarios de deforestación, con presencia y sin presencia de una nueva carretera asfaltada en el tramo dos (Huambé-Marsella) a lo largo del río Tigre. Asimismo, discutimos los impactos que generaría la nueva carretera y las alternativas existentes al poder negociar el carbono de las emisiones nacionales evitadas si esta carretera no se llevara a cabo. Finalmente, discutimos las oportunidades de la zona de estudio para el uso sostenible del territorio que asegure el mantenimiento de los bosques y turberas amazónicas en pie.

\section{MATERIAL Y MÉTODOS}

\section{ÁREA DE ESTUDIO}

Establecimos un área de influencia de $20 \mathrm{~km}$ alrededor de los 349,63 km de tramo de carretera que propone unir las comunidades de Huambé y Marsella, ubicadas en los distritos de San Juan Bautista y Tigre, en el departamento de Loreto (Provias Descentralizado, 2019). Esta área de influencia que va a lo largo del tramo propuesto cubre 1500405,21 hectáreas y representa el área de influencia promedio de las carreteras asfaltadas existentes en la Amazonía peruana, como la carretera Iquitos-Nauta en el departamento de Loreto y la carretera interoceánica en el departamento de Madre de Dios. La fisiografía del área de estudio es 


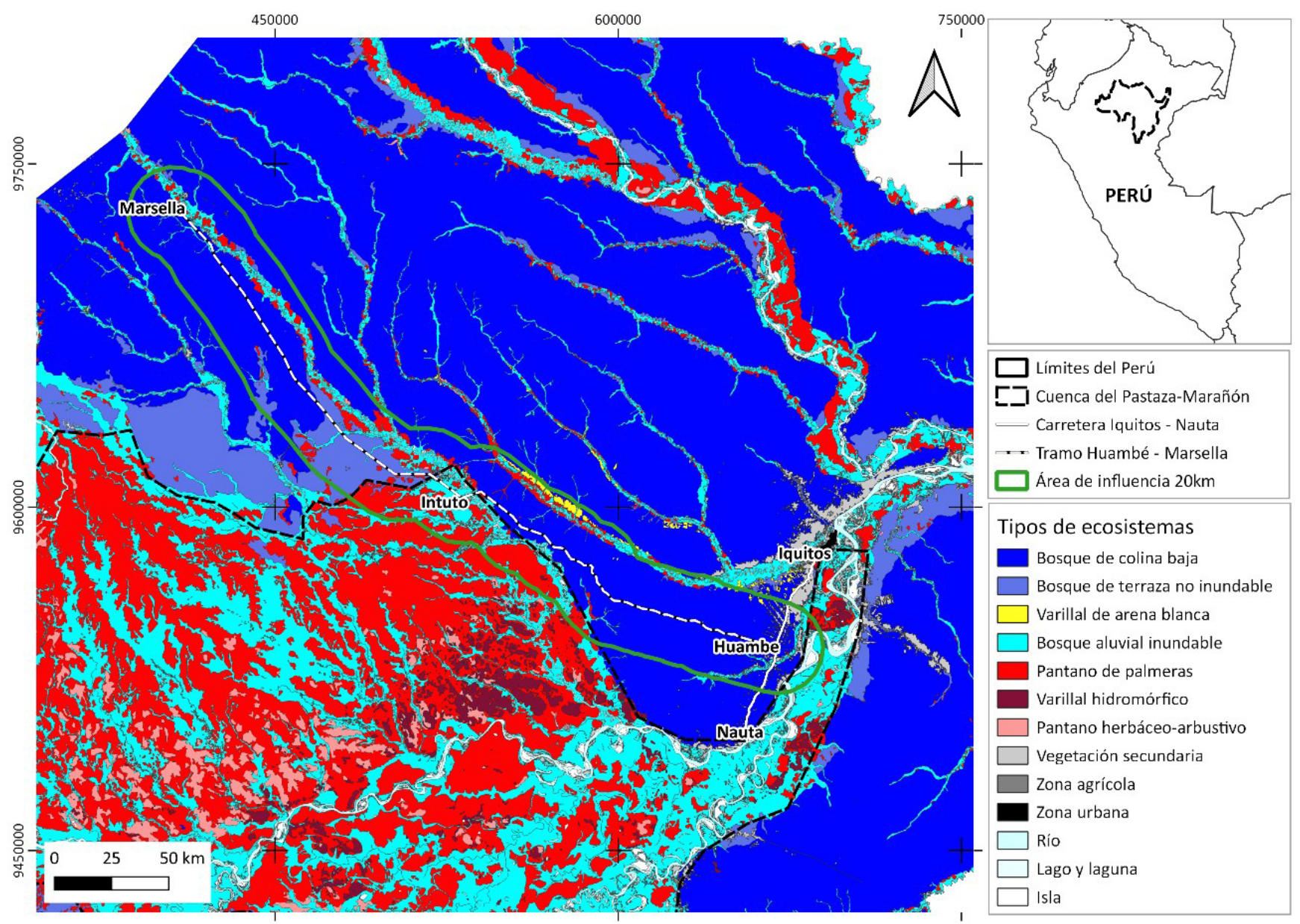

Figura 1. Tipos de ecosistemas en el área de influencia del tramo de carretera Huambé - Marsella a lo largo del río Tigre, Perú. El mapa de ecosistemas del MINAM (2019c) incluye el varillal hidromórfico de Draper et al. (2014).

principalmente ondulada, y existen diversos tipos de ecosistemas sobre colinas bajas, terrazas altas, aluvial inundable y turberas (Figura 1). El clima es cálido y húmedo, con 2,860 mm de precipitación anual y temperatura promedio máxima y mínima de $31,5^{\circ} \mathrm{C}$ y $21,9^{\circ} \mathrm{C}$, según los datos obtenidos del Servicio Nacional de Meteorología e Hidrología para la estación de Mazán para el periodo 1992-2013.

\section{COBERTURA Y PÉRDIDA DE BOSQUES}

Para obtener la cobertura y pérdida de bosques del área de estudio al 2018, utilizamos la plataforma de monitoreo de los cambios sobre la cobertura de los bosques peruanos - GeoBosques (MINAM, 2019a). Esta información es procesada por el Ministerio del Ambiente (MINAM), utilizando imágenes Landsat 8 de $30 \mathrm{~m}$ de resolución a partir del 2001 y con un mapa base de bosques/ no bosques de la Amazonía peruana al 2000.

Descargamos dos coberturas tipo "ráster" y extrajimos la información utilizando las áreas de influencia de $20 \mathrm{~km}$ definidas alrededor del tramo de la carretera Huambé-Marsella y de la carretera Iquitos-Nauta, esta última utilizada como escenario modelo de la presencia de una carretera asfaltada en Loreto (ver abajo). Del primer ráster denominado "Bosque y no bosque 2000 - Pérdida de bosque 2001-2018" extrajimos el área de la pérdida del bosque al 2000 (valor 
1 en el ráster), la cobertura de bosque al 2018 (valor 2), los cuerpos de agua (valor 3) y la pérdida total de bosque en el periodo 20012018 (valor 4; Figura 2). Del segundo ráster denominado "Pérdida de Bosque 2001-2018" extrajimos el área de pérdida anual de bosques (valores 1 al 18 en el ráster). El área de cada categoría fue obtenida en pixeles y fue convertida a hectáreas, multiplicando el valor por 0,09 debido a que el área de cada píxel fue de $30 \mathrm{~m} \mathrm{x}$ $30 \mathrm{~m}$.

\section{ESCENARIOS DE DEFORESTACIÓN}

Estimamos la tasa de cambio de la cobertura de bosque a no bosque (deforestación) del 2018 a los años 2030, 2046 y 2064 en base al área total del estudio (1 500 405,21 ha), usando dos escenarios de cambio:

a. Escenario habitual sin carretera o "business as usual" (BAU), donde asumimos que la tasa de deforestación de los bosques a lo largo del río Tigre incrementa linealmente en el tiempo. Para esto utilizamos el incremento lineal de la tasa de pérdida de bosques en el periodo 2001-2018 (Figura 3) y lo proyectamos al 2064.

b. Escenario con construcción de una carretera (CAR), donde asumimos que la tasa de deforestación de los bosques incrementa considerablemente siguiendo la tendencia de

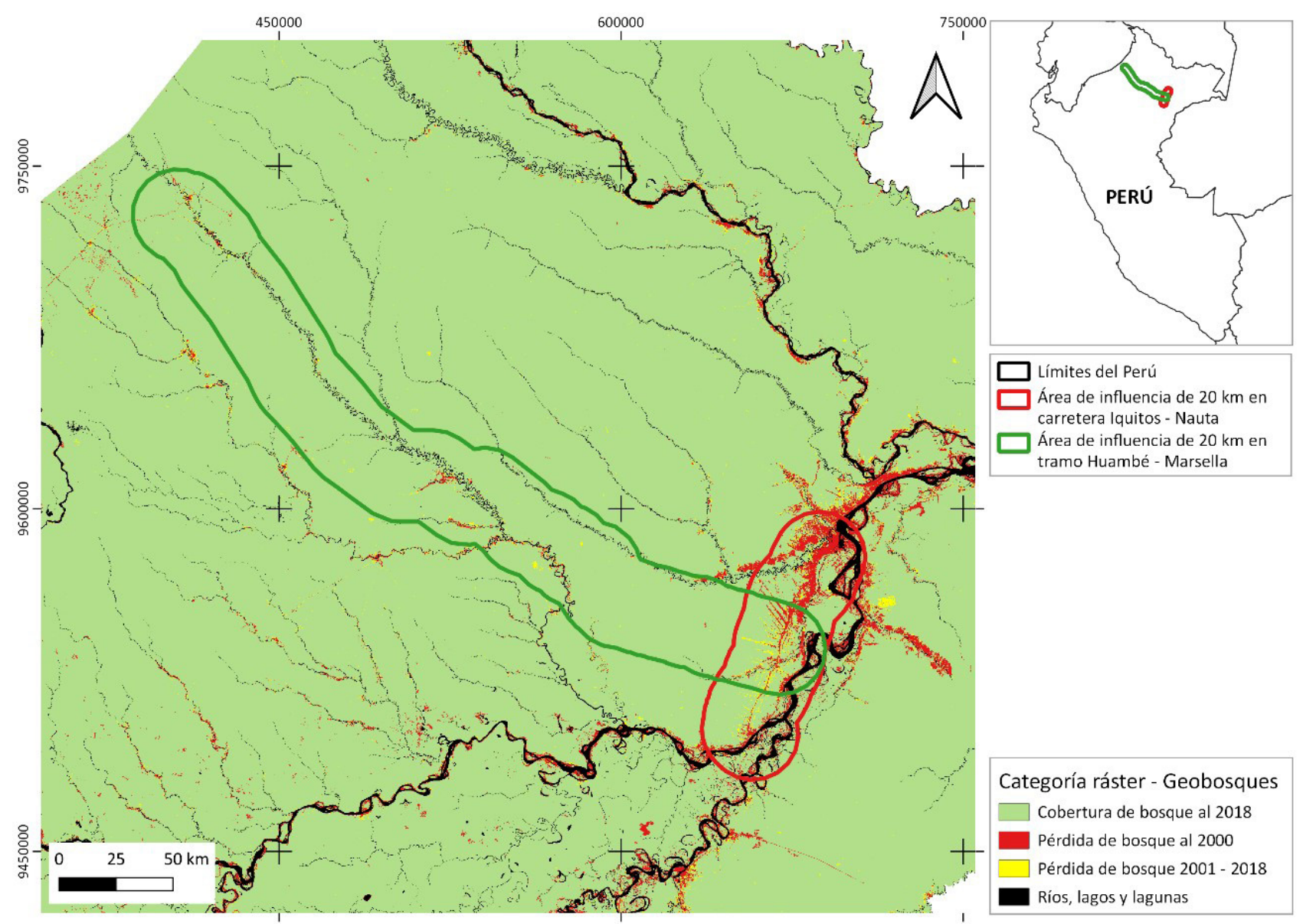

Figura 2. Cobertura y pérdida de bosques en las áreas de influencia del tramo de carretera Huambé-Marsella y de la carretera lquitos-Nauta, Loreto. 


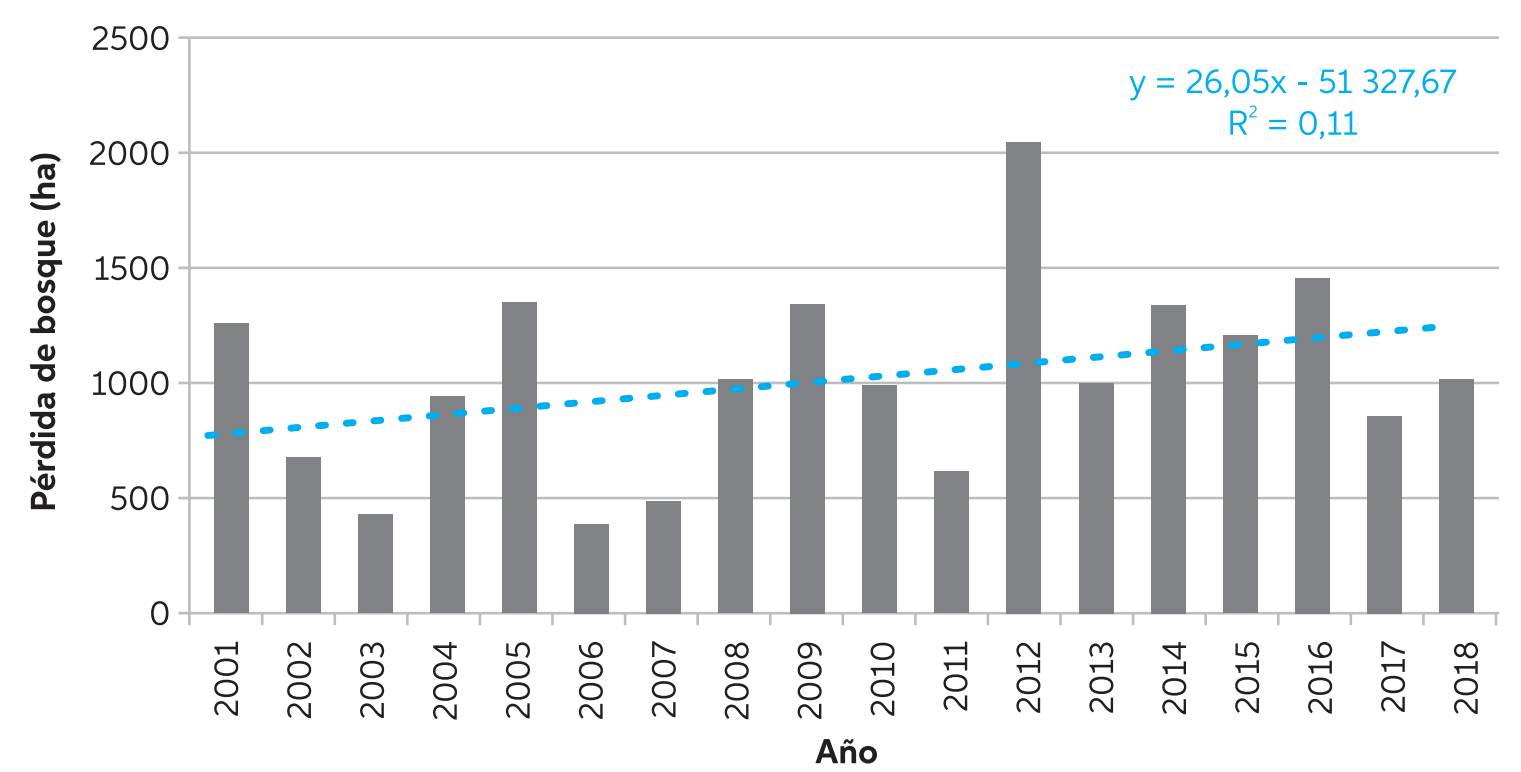

Figura 3. Pérdida anual de bosques registrada en la zona de influencia del tramo de carretera Huambé-Marsella a lo largo del río Tigre, Loreto. La línea azul muestra la relación lineal de pérdida de bosque en el periodo 2001-2018.

la deforestación en el área de influencia de 20 $\mathrm{km}$ alrededor de la carretera cercana existente Iquitos-Nauta (Mäki et al., 2001). Para esto, asumimos que el escenario en 1972 en la carretera Iquitos-Nauta, que corresponde al inicio de la construcción de la carretera, es el escenario de la zona de estudio al 2018, donde existe una pérdida de bosque concentrada principalmente en los extremos del tramo, en Huambé y Marsella. Asimismo, asumimos que los porcentajes de pérdida promedio anual de bosques en el área de influencia alrededor de la carretera Iquitos-Nauta en los periodos 19721999 (construcción de la carretera) y 20002018 (posterior a la construcción) representan las tasas esperadas de deforestación anual en la zona de influencia de $20 \mathrm{~km}$ alrededor de la carretera en estudio para los periodos 20182045 y $2046-2064$, respectivamente.

\section{PREDICCIÓN DE LA DEFORESTACIÓN}

Las áreas vulnerables a la pérdida de la cobertura de bosque (transición de bosque a no bosque) en la zona de estudio fueron determinadas a futuro utilizando el módulo Land Change Modeller (LCM) de Idrisi 17.0 The Selva (Eastman, 2012). Esto nos permitió estimar los porcentajes del área de los diferentes tipos de vegetación que se verían afectados. La transición fue modelada utilizando la red neuronal de perceptrones multicapas (MLP) y los mapas de cobertura de GeoBosques del 2000, 2014 y 2018 (MINAM, 2019a), considerando las categorías de bosque, no bosque y cuerpos de agua. Fueron utilizadas seis variables independientes con potencial para discriminar la vulnerabilidad a la deforestación, tales como la distancia a centros poblados (INEI, 2017), la distancia a ríos navegables (MINEDU, 2019), la distancia a carreteras primarias y secundarias (MTC, 2018), la distancia a áreas deforestadas al 2000, elevación y pendiente obtenidos del SRTM V2 (NASA, 2000).

El entrenamiento del modelo inició utilizando la información de los pixeles que experimentaron transición de bosque a no bosque en los mapas de cobertura del 2000 y 2014 y las variables 
independientes. Luego, el desempeño del modelo 2018 y comparando dicho modelo con el mapa real de cobertura de bosques de Geobosques al 2018 (MINAM, 2019a). El poder de predicción de las variables fue evaluado con el coeficiente de contingencia Cramer's V, mientras el rendimiento del método fue evaluado usando las curvas características de funcionamiento del receptor (ROC).

El modelamiento del potencial de transición al 2030, 2046 y 2064 se realizó utilizando los mapas de cobertura de bosques del 2000 y 2018 y las variables explicativas seleccionadas. Estas proyecciones incluyen la etapa de construcción de la carretera Huambé-Marsella al año del reporte de emisiones nacionales (2030), al año posterior a la construcción asumiendo que demorará un tiempo similar a la carretera Iquitos-Nauta (2046), y 18 años después que incluye el periodo total del presente estudio (2064). Las tasas de probabilidad de cambio de bosque a no bosque fueron ajustadas utilizando los valores de las tasas de cambio estimadas previamente para cada escenario (Tabla 1). En el caso del escenario fue evaluado proyectando la deforestación al

Las capas de otras categorías territoriales fueron recopiladas para estimar el porcentaje de deforestación futura que ocurriría dentro de las áreas naturales protegidas, áreas de conservación regional, zonas de amortiguamiento (SERNANP, 2019), comunidades indígenas, comunidades campesinas (DISAFILPA, 2019), concesiones forestales, bosques de producción (SERFOR, 2015) y concesiones petroleras (Petroperú, 2019). Todos los mapas fueron trabajados en QGIS (versión 3.10.2, A Coruña).

\section{ALMACENAMIENTO DE CARBONO}

El contenido de carbono de los diferentes tipos de vegetación fue estimado usando dos metodologías (Tabla 2). La primera está basada en el reporte nacional de gases de efecto invernadero del MINAM, donde la biomasa arriba del suelo incluye a todos los individuos con diámetro $\geq 10 \mathrm{~cm}, \mathrm{y}$ la biomasa debajo del suelo incluye el sistema radicular de los árboles (Biomasa debajo suelo $=0,489 \times$ Biomasa arriba del suelo^ 0,89 ) y no incluye el carbono orgánico del suelo y la turba (MINAM, 2019b). La biomasa total fue convertida a carbono multiplicando los valores por la fracción de carbono de 0,47. La segunda metodología está basada en parcelas forestales establecidas en los diferentes tipos de vegetación de Loreto (Draper et al., 2014; Honorio et al., 2015; Sullivan et al., 2017; Bhomia et al., 2018) y contiene nuevos muestreos realizados en la zona de estudio (Honorio et al., 2020). Incluye la biomasa aérea y radicular de todos los CAR se incluyó un incentivo de $5 \mathrm{~km}$ alrededor de la nueva carretera propuesta dándole valor de 1,5 , siendo esta área preferida para el cambio del resto de los pixeles del área de estudio. individuos con diámetro $\geq 10 \mathrm{~cm}$ y el carbono orgánico del suelo a $2 \mathrm{~m}$ de profundidad y en la turba. La biomasa arriba del suelo fue estimada usando 16 parcelas establecidas en pantanos de 
Tabla 2. Contenido de carbono arriba y debajo del suelo en los diferentes tipos de vegetación reportados en el área de influencia del tramo de carretera Huambé-Marsella.

\begin{tabular}{|c|c|c|c|c|}
\hline Tipo de vegetación & Area* (ha) & $\begin{array}{l}\text { Carriba del } \\
\left.\text { suelo (t } C \text { ha }^{-1}\right)\end{array}$ & $\begin{array}{l}\text { C debajo del } \\
\left.\text { suelo (t } C \text { ha }^{-1}\right)\end{array}$ & $\begin{array}{l}\text { Carbono total } \\
\text { (Mt C) }\end{array}$ \\
\hline \multicolumn{5}{|c|}{ ( $\alpha$ ) Usando valores de biomasa arbórea (MINAM, 2019b) } \\
\hline Bosque de colina baja & 915066,13 & 111,97 & 32,59 & 132,28 \\
\hline B. de terraza no inundable & 122996,19 & 111,97 & 32,59 & 17,78 \\
\hline Varillal de arena blanca & 11511,76 & 111,97 & 32,59 & 1,66 \\
\hline Bosque aluvial inundable & 188234,10 & 67,39 & 20,74 & 16,59 \\
\hline Pantano de palmeras & 170646,80 & 67,39 & 20,74 & 15,04 \\
\hline Varillal hidromórfico & 1052,72 & 67,39 & 20,74 & 0,09 \\
\hline Pantano herb.-arbustivo & 723,71 & 67,39 & 20,74 & 0,06 \\
\hline TOTAL & 1410231,42 & & & 183,51 \\
\hline \multicolumn{5}{|c|}{ ( $\beta$ ) Usando valores de biomasa arbórea y carbono en suelo y turba medidos en parcelas forestales en Loreto } \\
\hline Bosque de colina baja ${ }^{3,5}$ & 915066,13 & 125,04 & 130,72 & 234,04 \\
\hline B. de terraza no inundable ${ }^{3}$ & 122996,19 & 125,04 & 130,72 & 31,46 \\
\hline Varillal de arena blanca ${ }^{3}$ & 11511,76 & 114,61 & 119,82 & 2,70 \\
\hline Bosque aluvial inundable 2 & 188234,10 & 90,02 & 94,11 & 34,66 \\
\hline Pantano de palmeras s.,4,5 $^{1,4}$ & 170646,80 & 85,66 & 929,22 & 173,19 \\
\hline Varillal hidromórfico, ${ }^{1,4,5}$ & 1052,72 & 85,49 & $1,567,24$ & 1,74 \\
\hline Pantano herb.-arbustivo ${ }^{1}$ & 723,71 & 0,00 & 976,32 & 0,71 \\
\hline TOTAL & 1410231,42 & & & 478,49 \\
\hline
\end{tabular}

*El área restante está representada por cuerpos de agua (40 694,31 ha), zona agrícola y vegetación secundaria (49 479,48 ha).

${ }^{1}$ Draper et al. (2014), ${ }^{2}$ Honorio et al. (2015), ${ }^{3}$ Sullivan et al. (2017), ${ }^{4}$ Bhomia et al. (2018) y ${ }^{5}$ Honorio et al. (2020).

palmeras, 15 en varillales hidromórficos, nueve en bosques aluviales inundables, ocho en bosques de colina baja y terrazas no inundables y cuatro en los varillales de arena blanca. Asumimos que el pantano herbáceo-arbustivo no presenta árboles con diámetro $\geq 10 \mathrm{~cm}$ y por lo tanto su biomasa aérea fue cero (Draper et al., 2014). La biomasa fue multiplicada por 0,47 para obtener el contenido de carbono arriba del suelo. El carbono debajo del suelo en las turberas se obtuvo al multiplicar la profundidad de la turba ( $\mathrm{n}=298$ puntos de muestreo), el contenido de carbono y la densidad aparente $(\mathrm{n}=31$ sitios) para cada ecosistema de turbera, obteniendo valores promedio de $1,86 \mathrm{~m}, 37 \%$ y $0,13 \mathrm{~g} \mathrm{~cm}^{-3}$ para el pantano de palmeras, 3,22 m, $50 \%$ y $0,10 \mathrm{~g} \mathrm{~cm}^{-3}$ para el varillal hidromórfico, y $2,74 \mathrm{~m}, 46 \%$ y $0,06 \mathrm{~g} \mathrm{~cm}^{-3}$ para el pantano herbáceo-arbustivo, respectivamente (Draper et al., 2014; Bhomia et al., 2018; Honorio et al., 2020). El carbono debajo del suelo de los bosques sin turba fue estimado como un porcentaje del carbono total, representando el carbono arriba del suelo el $48,9 \%$, el sistema radicular el 10,8 \% y el carbono a 2 m de profundidad del suelo el 40,3\% (Malhi et al., 2009).

\section{EMISIONES DE CO,}

El área potencial de transición de bosques a no bosques en hectáreas estimada para cada 
periodo (2019-2030, 2019-2046 y 2019-2064) fue distribuida por tipo de vegetación utilizando el mapa de ecosistemas (MINAM, 2019c), al cual le integramos una capa de la distribución de los varillales hidromórficos (Draper et al., 2014) que fueron recientemente descritos para la ciencia (Draper et al., 2018). Luego, cada área fue convertida a emisiones de carbono, multiplicando los valores por el carbono total de cada tipo de vegetación y por el factor $44 / 12$ para convertir el carbono en unidades de $\mathrm{CO}_{2}$ equivalente (IPCC, 2006). Por lo tanto, los valores de las emisiones representan las emisiones de $\mathrm{CO}_{2}$ brutas potenciales de la deforestación y no incorporan las emisiones de otros gases de efecto invernadero, ni el impacto de la regeneración de los bosques ni las reservas de carbono a las opciones de posteriores usos del suelo.

\section{RESULTADOS}

\section{ESCENARIOS DE DEFORESTACIÓN}

La pérdida acumulada de bosques al 2018 en el área de estudio fue de 3,4\% o 49 479,48 ha (Figura 2), mostrando una tasa de pérdida creciente en los últimos 18 años, pasando de un promedio de pérdida anual de 837 ha en 2001-2006 a 1141 hectáreas en 2013-2018, con una mayor pérdida de bosques (2,043 ha) ocurrida en el 2012 (Figura 3). Proyectando una tasa de deforestación lineal, se estima que la pérdida acumulada de bosques al 2064 ascendería a 9,2 \% o 134 686,81 ha en el escenario BAU, mientras la pérdida ascendería a $25,5 \%$ o 372226,28 ha en el escenario CAR (Figura 4). Las tasas de cambio resultantes del 2018 al 2030, 2046 y 2064 variaron de 0,0113 a

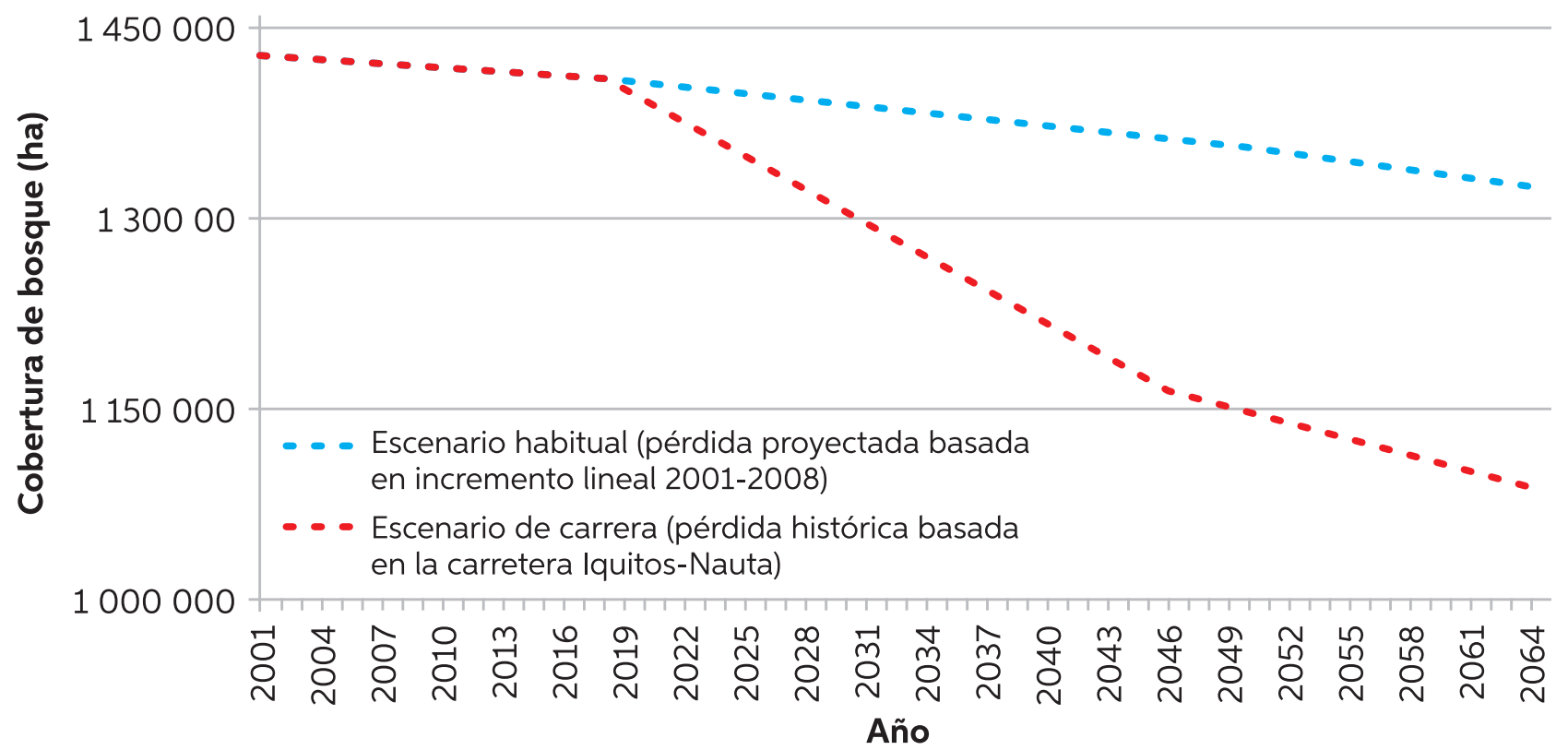

Figura 4. Cobertura de bosques en el área de influencia del tramo de carretera Huambé-Marsella bajo dos escenarios de deforestación, escenario habitual (BAU) y escenario con carretera (CAR). Los valores del periodo 2001-2018 fueron tomados de GeoBosques, mientras los valores en los siguientes 46 años fueron estimados utilizando: (a) el escenario BAU que estima una pérdida lineal de bosque constante en el tiempo, y (b) el escenario CAR que estima una pérdida según los datos históricos de deforestación de la carretera cercana existente lquitos-Nauta. 
0,0568 en el escenario BAU y de 0,0705 a 0,2151 en el escenario CAR (Tabla 1).

\section{PREDICCIÓN DE LA DEFORESTACIÓN}

El desempeño del modelo al comparar los mapas de deforestación proyectada y original al 2018, mostró una tasa de precisión de 85,90\% utilizando las seis variables ( $\mathrm{ROC}=0,959 ; 41,697$ falsas alarmas, 47869 fallas y 2305 éxitos) y de $86,24 \%$ excluyendo la pendiente $(\mathrm{ROC}=0,959$; 41894 falsas alarmas, 48066 fallas y 2108 éxitos). La pendiente fue la única variable excluida del análisis por presentar valor de Cramer's $\mathrm{V}$ de 0,0086 , mientras las otras variables mostraron valores mayores a 0,20 , es decir, fueron variables significativas y explicativas de la predicción de la transición bosque a no bosque al 2018.

El modelamiento estimó una pérdida de bosques de 15 923,25 ha, 42569,37 ha y 80071,11 ha para el escenario BAU en los periodos 20192030, 2019-2046 y 2019-2064, respectivamente, mientras estos valores fueron 99385,83 ha, 231910,83 ha y 303 248,79 ha en el escenario CAR para los mismos periodos (Tabla 3a y Figura 5). Los tipos de vegetación más vulnerables a la deforestación serían el bosque de colina baja y el bosque aluvial inundable, seguidos del bosque de palmeras, mientras las categorías territoriales más afectadas serían los territorios de las comunidades indígenas y lotes petroleros, seguidos por los bosques de producción permanente y las concesiones forestales (Tabla 4).

\section{ALMACENAMIENTO DE CARBONO}

El carbono almacenado arriba del suelo varió entre los diferentes tipos de vegetación, siendo los valores del MINAM generalmente más bajos (67,39-111,97 t C ha-1) que los valores estimados utilizando las parcelas forestales por tipo de vegetación $(85,49-125,04$ t C ha-1; Tabla 2). El almacenamiento de carbono debajo del suelo varió bastante dependiendo del método utilizado, incrementando 4 a 5 veces los valores al contabilizar el carbono orgánico en el suelo en bosques sin turba y de 45 a 76 veces más al considerar la turba en los ecosistemas de turbera. La reserva total de carbono en la zona de estudio fue estimada en 183,51 millones de toneladas de carbono (Mt C) utilizando el método del MINAM, siendo el bosque de colina baja el que más contribuyó, debido a su mayor extensión. La reserva fue de 478,49 Mt C cuando se incluyó además el carbono almacenado en el suelo y la turba, siendo el bosque de colina baja y el pantano de palmeras los que más contribuyeron (Tabla 2).

\section{EMISIONES DE CO,}

Utilizando los valores de biomasa arbórea del MINAM, las pérdidas futuras de cobertura de bosque para el periodo 2019-2064 generarían emisiones brutas de 35,55 millones de toneladas de $\mathrm{CO}_{2}$ equivalentes ( $\mathrm{Mt} \mathrm{CO}_{2}$-eq) y 135,56 $\mathrm{Mt} \mathrm{CO}_{2}$ eqpara los escenarios BAUy CAR, respectivamente (Tabla 3a). La no construcción de la carretera podría evitar la emisión de 41,49 Mt $\mathrm{CO}_{2}$-eq a la atmósfera en el periodo 2019-2030. La inclusión del carbono en el suelo y la turba en los cálculos incrementó las emisiones totales entre 1,9 a 2,6 veces dependiendo del periodo y escenario evaluado (Tabla $3 \mathrm{~b}$ ).

\section{DISCUSIÓN}

IMPACTOS DE LA CONSTRUCCIÓN DE LA CARRETERA

El nuevo proyecto de carretera Huambé -Marsella, a lo largo del río Tigre en el departamento de Loreto, tendrá un alto impacto ambiental al generar una pérdida adicional de cobertura de bosques de 83 462,58 hectáreas para el periodo 2019-2030. Esta pérdida es mayor a la estimada por un estudio reciente para toda la carretera Iquitos-Saramiriza en una zona de influencia de 

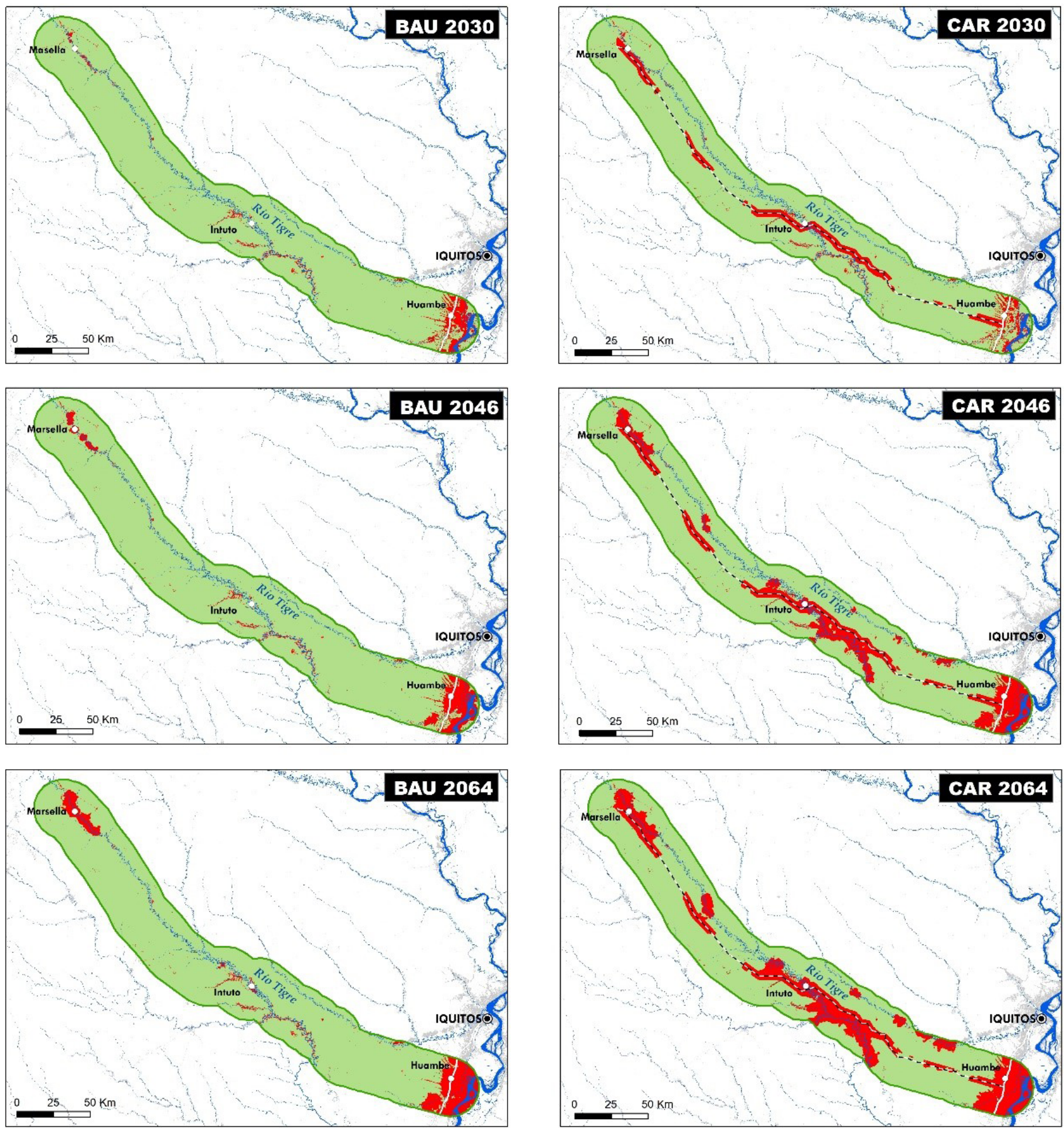

OCapital de departamento .... Tram

Figura 5. Deforestación proyectada al 2030, 2046 y 2064 en el área de influencia del tramo de carretera Huambé-Marsella bajo dos escenarios de deforestación, escenario habitual (BAU) y escenario con carretera (CAR). 


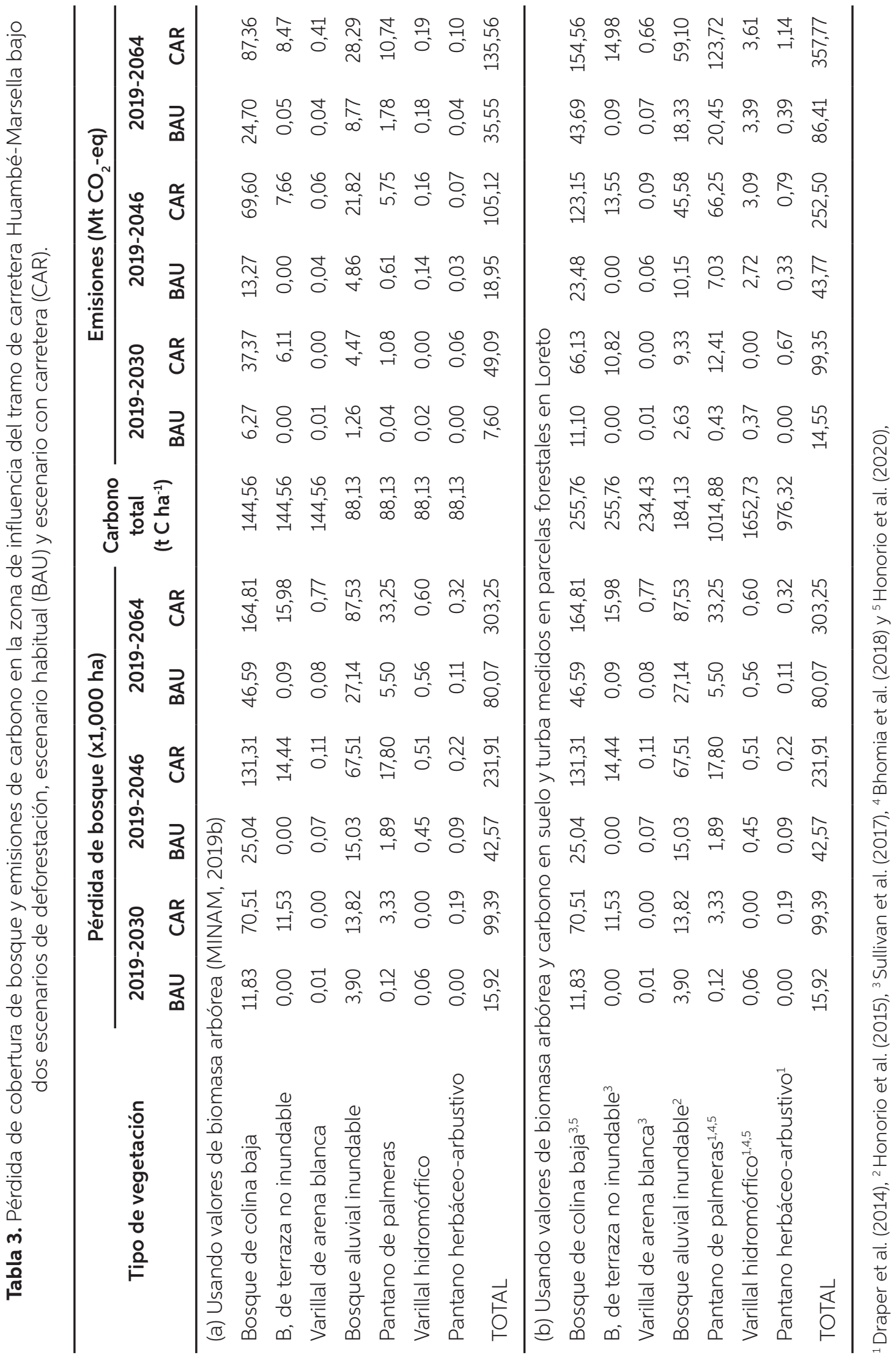


Tabla 4. Pérdida de bosques según tipos de vegetación y categorías territoriales en la zona de influencia del tramo de carretera Huambé-Marsella bajo dos escenarios de deforestación, escenario habitual (BAU) y escenario con carretera (CAR).

\begin{tabular}{lrrrrrr} 
& \multicolumn{7}{c}{ Pérdida de bosques (\%) } \\
\cline { 2 - 7 } Tipos de vegetación & \multicolumn{1}{c}{$2019-2030$} & $2019-2046$ & \multicolumn{2}{c}{$2019-2064$} \\
& BAU & CAR & BAU & CAR & BAU & CAR \\
\hline Bosque de colina baja & 74,32 & 70,95 & 58,82 & 56,62 & 58,19 & 54,35 \\
B. de terraza no inundable & 0,00 & 11,60 & 0,00 & 6,23 & 0,12 & 5,27 \\
Varillal de arena blanca & 0,07 & 0,00 & 0,16 & 0,05 & 0,10 & 0,25 \\
Bosque aluvial inundable & 24,49 & 13,90 & 35,31 & 29,11 & 33,90 & 28,87 \\
Pantano de palmeras & 0,72 & 3,36 & 4,44 & 7,68 & 6,86 & 10,96 \\
Varillal hidromórfico & 0,39 & 0,00 & 1,05 & 0,22 & 0,70 & 0,20 \\
Pantano herbáceo-arbustivo & 0,01 & 0,19 & 0,22 & 0,10 & 0,14 & 0,11 \\
\hline Categorías territoriales & $2019-2030$ & $2019-2046$ & $2019-2064$ \\
Áreas naturales protegidas & BAU & CAR & BAU & BAU & CAR & BAU \\
Zonas de amortiguamiento & 1,18 & 0,80 & 0,51 & 0,05 & 0,18 & 0,13 \\
Áreas de conservación regional & 0,00 & 0,00 & 0,00 & 5,73 & 3,30 & 4,04 \\
Comunidades indígenas & 3,19 & 2,50 & 2,25 & 0,16 & 0,85 & 1,33 \\
Comunidades campesinas & 3,50 & 10,18 & 17,09 & 40,76 & 36,46 & 39,54 \\
Bosques producción permanente & 0,01 & 0,00 & 0,12 & 0,00 & 1,00 & 2,33 \\
Concesiones forestales & 0,00 & 0,01 & 0,71 & 20,69 & 14,44 & 12,67 \\
Lotes petroleros & 0,00 & 0,21 & 0,79 & 14,74 & 9,83 & 8,26 \\
Todas las unidades combinadas & 3,92 & 15,66 & 21,81 & 10,87 & 9,73 & 10,28 \\
& 8,91 & 21,65 & 32,71 & 64,86 & 57,96 & 62,55 \\
\hline
\end{tabular}

$20 \mathrm{~km}$ al 2030 (35 961 ha; Vilela et al., 2020). Dicho estudio modeló la deforestación utilizando datos de cobertura de bosque y no bosque del 2011 y 2015 y variables similares promotoras de la deforestación. La baja tasa de deforestación obtenida en dicho estudio es posible que se deba a la dificultad existente para modelar a futuro la trayectoria en la colonización del territorio en la Amazonía, ya que esta tarea involucra variables externas como el tráfico ilegal de la tierra, la promoción esporádica de la agricultura por los gobiernos y la creciente demanda de los recursos naturales en bosques antes inaccesibles (Foley et al., 2007; Laurance et al., 2009). Por este motivo, nuestro modelamiento de la deforestación en el escenario con carretera incluyó la tasa de cambio ocurrida en la cercana carretera asfaltada IquitosNauta donde el cambio del uso del suelo forestal para la expansión agrícola y urbana ocurrió sin planificación del territorio (Mäki et al., 2001), llegando a perder el $25,5 \%$ de la cobertura de bosques al 2018.

La construcción de esta nueva carretera a lo largo del río Tigre es una amenaza no solo para la conservación de una de las áreas más extensas de bosque tropical del planeta (Watson et al., 2018), 
sino también para la conservación de las turberas más profundas de la cuenca amazónica (Baker et al., 2019). En particular, la turbera más profunda se ubica en Aucayacu, en el río Tigre, donde se ha reportado una capa de materia orgánica de 7,45 metros de profundidad que empezó a formarse hace 8000 años (Lähteenoja \& Page, 2011). En la zona de estudio, los varillales hidromórficos, los pantanos de palmeras y los pantanos herbáceoarbustivos son los ecosistemas característicos con presencia de turba (Lähteenoja et al., 2012; Draper et al., 2014; Bhomia et al., 2019; Honorio et al., 2020), mostrando una pérdida potencial total de 6165,31 ha en el escenario BAU y 34162,32 ha en el escenario CAR del 2019-2064 y siendo los pantanos de palmeras los más afectados. Las turberas amazónicas son actualmente vulnerables al cambio del uso de la cobertura de bosque debido a la expansión del cultivo de la palma aceitera y del arroz (Roucoux et al., 2017; Vijay et al., 2018), siendo la pérdida del carbono en turberas irreparable (Goldstein et al., 2020). Por lo tanto, estos resultados resaltan la importancia en la conservación y manejo sostenible de las turberas en la Amazonía peruana para asegurar la permanencia de la vegetación y las condiciones hidrológicas que promueven la acumulación de la turba.

Esta nueva carretera también fue categorizada sin retorno económico positivo debido al alto costo de inversión y mantenimiento, así como a su alto impacto socioambiental (Vilela et al., 2020). Esto incluye el impacto directo en el bienestar de las poblaciones locales, al reducir los servicios ecosistémicos de los bosques (provisión de alimentos, caza, ambientes culturales, otros), la generación de nuevos conflictos sociales relacionados a actividades ilegales (tala y minería ilegal) y el incremento de la abundancia de insectos que son vectores de enfermedades endémicas como la malaria (Vittor et al., 2006; Baker et al., 2019; Martín et al., 2019). Los impactos generados por las carreteras no son ajenos en la Amazonía peruana. Se cuenta con algunos ejemplos que evidencian las consecuencias que tiene la construcción de carreteras en los bosques amazónicos peruanos. La carretera interoceánica, en el departamento de Madre de Dios, generó la pérdida de la conectividad de los bosques, incrementándose además la minería ilegal (Sánchez Cuervo et al., 2020); en el departamento de Ucayali, la carretera Jorge Basadre favoreció el incremento de la tala ilegal de madera, las plantaciones de palma aceitera y los cultivos ilegales de coca (Hyman \& Barona, 2010); y en el departamento de Loreto, la carretera Iquitos-Nauta, permitió el acceso a ecosistemas frágiles como los varillales de arena blanca, donde la extracción de arena para la construcción ha generado graves problemas ambientales (Mäki et al., 2001). No queda duda que el principal impulsor de la deforestación y degradación de los bosques amazónicos peruanos es la apertura de nuevas carreteras y los departamentos con extensas áreas de bosques y turberas como Loreto son vulnerables a dichos impactos.

\section{ALTERNATIVAS PARA LA MITIGACIÓN DE EMISIONES}

El Perú viene trabajando en la identificación de nuevas políticas y programas de financiamiento para reducir las emisiones nacionales de gases de efecto invernadero a través de la conservación de los bosques y el manejo sostenible de los recursos. Por ejemplo, tenemos el programa de reducción de emisiones por deforestación y degradación forestal (REDD; Ochieng et al., 2018), el fondo verde para el clima (GCF; Roucoux et al., 2017) y las medidas de adaptación y mitigación para reducir las emisiones determinadas nacionales (NDC; Vazquez-Rowe et al., 2019). Sin embargo, nuevas medidas tienen que ser consideradas para la reducción de la deforestación en la 
Amazonía peruana. Por ejemplo, no construir la carretera junto al río Tigre evitaría la pérdida de 83 462,58 hectáreas de bosque y la emisión de 41,49 Mt $\mathrm{CO}_{2}$-eq del 2019-2030. Estas emisiones evitadas son equivalentes al 1,31\% de las emisiones nacionales previstas durante el mismo período o al $13,91 \%$ de las emisiones nacionales previstas en 2030 (2030: 298,30 Mt CO2-eq; GTM-NDC, 2018). No construir la carretera también evitaría las emisiones de gases vinculadas al nuevo tráfico que se generaría y a las materias primas utilizadas durante la construcción de una nueva carretera, emisiones que no han sido cuantificadas en el presente estudio pero que causarían también impactos ambientales y en la salud de las poblaciones locales (Larrea-Gallegos et al., 2017). El costo para construir la carretera, que asciende a US \$ 1067,2 millones (US\$ 3,052,370 por km; Vilela et al., 2020), podría alternativamente utilizarse para financiar proyectos de mejora del transporte fluvial utilizando embarcaciones modernas, económicas y ecológicas (Gallice et al., 2019) que correspondan a las características de los ríos de Loreto, con la mejora de puertos, embarcaderos y el monitoreo del canal de navegación. Asimismo, un servicio de aerotaxis podría atender las necesidades de los pobladores indígenas y ribereños. La correcta mejora del transporte en la Amazonía para evitar la deforestación podría ser una medida de mitigación de emisiones propuesta en las NDC de Perú.

Otra alternativa de mitigación de emisiones podría incluir la conservación y manejo sostenible de las turberas amazónicas, en las que los depósitos de turba almacenados debajo del suelo contribuyen en gran medida al almacenamiento de carbono de los bosques amazónicos. La turba se acumula en condiciones de baja concentración de oxígeno en humedales con permanente saturación de agua en el suelo, donde la tasa de descomposición de la materia orgánica es baja y supera la tasa de producción (Lähteenoja et al., 2012). El hallazgo de que grandes cantidades de carbono se almacenan debajo del suelo en las turberas de Loreto (Draper et al., 2014) fue crucial para apoyar una propuesta exitosa dirigida por el Fondo de Promoción de las Áreas Naturales Protegidas del Perú al GCF (Roucoux et al., 2017). Este proyecto, que comenzó en agosto del 2017, trabaja con comunidades indígenas para establecer bionegocios basados en la comercialización de frutos de palmeras que se aprovechan de manera sostenible a través de la escalada, evitando la muerte de las palmeras. Este proyecto tiene como objetivo mejorar el manejo y la conservación de 343000 hectáreas de turberas y bosques en 120 comunidades en la provincia de Datém del Marañón en el departamento de Loreto.

En la zona de estudio a lo largo del río Tigre, se almacenan 162,77 Mt C arriba del suelo, con un almacén adicional de más de 315,72 Mt C debajo del suelo, cuando se incluye el carbono del suelo y la turba. En particular, se almacenan 1014,88 t C ha ${ }^{-1}$ en pantanos de palmeras, 1652,73 t C ha $^{-1}$ en varillales hidromórficos y 976,32 t C ha-1 en pantanos abiertos (Draper et al., 2014; Bhomia et al., 2018; Honorio et al., 2020). Evitar la deforestación de una hectárea de estas turberas podría generar un ingreso promedio de US \$ 4454 en el mercado de carbono, considerando el precio de US \$ 1 por tonelada evitada de emisiones de $\mathrm{CO}_{2}$-eq. Este valor es mucho más alto que el valor de US \$ 323 por hectárea estimado si solo se incluye la biomasa de los árboles según el inventario nacional de GEI (MINAM, 2019b). Por lo tanto, reconocer e incluir el carbono almacenado en el suelo y la turba en las estimaciones de las emisiones nacionales es importante porque existe un gran potencial para conservar y manejar sosteniblemente los bosques y turberas amazónicas para cumplir con los compromisos en la mitigación del cambio 
climático a través de medidas NDC o proyectos REDD y GCF.

\section{POTENCIALIDADES PARA LA CONSERVACIÓN Y MANEJO SOSTENIBLE EN EL RIO TIGRE}

La presencia de comunidades indígenas a lo largo del río Tigre presenta una ventaja al desarrollo de proyectos de conservación y manejo, debido al bajo impacto que ejercen las comunidades sobre los bosquesy a losvalores culturales que favorecen la conservación en esta parte de la Amazonía peruana (Honorio et al., 2020). Los bosques y sus recursos están estrechamente relacionados a la cultura de los pueblos indígenas amazónicos, generando medidas de protección y respeto que favorecen su conservación (Schultz et al., 2019). Por ejemplo, el pueblo urarina adaptado a vivir en los humedales de la cuenca del río Chambira, confecciona el "ela" o "cachihuango" que es un tejido tradicional elaborado con las hojas jóvenes del aguaje, cuyo conocimiento es transmitido de generación en generación entre las mujeres (Martín et al., 2019). Las comunidades a lo largo del río Tigre cuentan con 170 646,80 hectáreas de pantanos de palmeras en la zona de estudio. El manejo sostenible de las plantas femeninas de aguaje no solo aseguraría la supervivencia de la especie, sino que también podría generar ingresos a las comunidades y así mantener la biomasa y el carbono de los bosques. El manejo del aguaje podría ser también una alternativa para empoderar a las comunidades en la protección de sus bosques y reducir los conflictos históricos con las concesiones petroleras y concesiones forestales (Baker et al., 2019), con quienes solo han conseguido la contaminación del ambiente por derrames de petróleo y el incremento de la tala ilegal (Díaz-Alván et al., 2017).

En la zona de estudio también existen 1052,72 hectáreas de varillales hidromórficos que son ecosistemas de turberas con la mayor densidad de carbono almacenada por unidad de área de la cuenca amazónica (Draper et al., 2014). A pesar de ubicarse dentro del Abanico del Pastaza, la falta de un instrumento de gestión para el adecuado manejo y conservación de este sitio Ramsar pone en riesgo la conservación de estos ecosistemas. Urge establecer medidas de conservación adecuadas en esta zona para proteger el carbono, las plantas y los animales especialistas de hábitats de bajos nutrientes que albergan estos ecosistemas (Díaz-Alván et al., 2017; Draper et al., 2018). Asimismo, la presencia de turba fue una información relevante para la creación del Parque Nacional Yaguas donde se conservan más de 800,000 hectáreas de bosques en la cuenca baja del río Putumayo en Loreto. No tenemos que esperar la presencia de una nueva carretera para buscar mecanismos de conservación efectivos o crear un área protegida, como fue el caso de la Reserva Nacional Allpahuayo-Mishana, creada en 1999 como refugio para la fauna silvestre y la conservación de ecosistemas frágiles como los varillales de arena blanca y bosques inundables por aguas negras (Salo \& Pyhälä, 2007).

\section{CONCLUSIONES}

Este estudio revela el gran impacto ambiental, social y económico que el tramo de carretera propuesto para unir la comunidad de Huambé con la de Marsella tendría sobre la cobertura de los bosques y turberas y las comunidades locales que viven a lo largo del río Tigre. Conservar los bosques y turberas en pie ayudaría a mantener el bienestar de las poblaciones locales y los compromisos internacionales ante el cambio climático. Por lo tanto, evitar la construcción de la carretera a lo largo del río Tigre debería compensarse con otras alternativas como la mejora del transporte fluvial, servicio de aerotaxi e impulsando la conservación y manejo sostenible de las turberas, que podrían ser considerados 
como mecanismos viables de mitigación de las emisiones de gases de efecto invernadero a la atmósfera en el Perú.

\section{AGRADECIMIENTOS}

Este trabajo fue posible gracias al financiamiento de la Wildlife Conservation Society (WCS) y realizado bajo convenio entre WCS y el Instituto de Investigaciones de la Amazonía Peruana (IIAP).

\section{REFERENCIAS BIBLIOGRÁFICAS}

Asner, G.P.; Knapp, D.E.; Martin, R.E.; Tupayachi, R.; Anderson, C.B.; Mascaro, J.; Sinca, F.; Chadwick, K.D.; Sousan, S.; Higgins, M.; Farfan, W.; Silman, M.R.; Llactayo León, W.A.; Neyra Palomino, A.F. 2014. La geografía del carbono en alta resolución del Perú. Observatorio Aéreo Carnegie, Ministerio del Ambiente del Perú. Lima, Perú. 64pp.

Baker, T.R.; Del Castillo Torres, D.; Honorio Coronado, E.N.; Lawson, I.; Martín Brañas, M.; Montoya, M.; Roucoux, K.H. 2019. The challenges for achieving conservation and sustainable development within the wetlands of the Pastaza-Marañón basin, Peru. In: Chirif A. (Ed.). Peru: Deforestation in times of climate change. p. 155-174.

Bhomia, R.K.; Van Lent, J.; Rios, J.M.G.; Hergoualc'h, K.; Honorio Coronado, E.N.; Murdiyarso, D. 2018. Impacts of Mauritia flexuosa degradation on the carbon stocks of freshwater peatlands in the Pastaza-Marañón river basin of the Peruvian Amazon. Mitigation and adaptation strategies for global change, 24(4): 645-668. DOI: https://doi.org/10.1007/s11027-0189809-9
Blackman, A.; Corral, L.; Lima, E.S.; Asner, G.P. 2017. Titling indigenous communities protects forests in the Peruvian Amazon. Proceedings of the National Academy of Sciences, 114(16): 4123-4128. DOI: https://doi.org/10.1073/ pnas. 1603290114

Blanco-Gutiérrez, I.; Manners, R.; Varela-Ortega, C.; Tarquis, A.; Martorano, L.G.; Toledo, M. 2019. Examining the sustainability and development challenge in agricultural-forest frontiers of the Amazon Basin through the eyes of locals. Natural Hazards and Earth System Sciences: 1-35. DOI: https://doi.org/10.5194/ nhess-20-797-2020

Díaz-Alván, J.; Socolar, J.B.; Álvarez Alonso, J. 2017. The avifauna of the Río Tigre basin, northern Perú. Ornitologia Neotropical, 28: 11-21.

DISAFILPA, 2019. Mapa de comunidades indígenas y campesinas del departamento de Loreto. Dirección de Saneamiento Físico Legal de la Propiedad Agraria de Loreto. (http:// siar.minam.gob.pe/loreto/mapas/mapacomunidades -indigenas-departamentoloreto). Acceso: 12/06/2020.

Draper, F.C.; Roucoux, K.H.; Lawson, I.T.; Mitchard, E.T.; Honorio Coronado, E.N.; Lähteenoja, O.; Montenegro, L.T.; Sandoval, E.V.; Zaráte, R.; Baker, T.R. 2014. The distribution and amount of carbon in the largest peatland complex in Amazonia. Environmental Research Letters, 9: 124017. DOI: https://doi.org/10.1088/17489326/9/12/124017

Draper, F.C.; Honorio Coronado, E.N.; Roucoux, K.H.; Lawson, I.T.; Pitman, N.C.A.; Fine, P.V.A.; Phillips, O.L.; Torres Montenegro, L.A.; Valderrama Sandoval, E.; Mesones, I.; GarcíaVillacorta, R.; Ramirez Arévalo, F.R.; Baker, T.R. 2018. Peatland forests are the least diverse tree communities documented in Amazonia, but contribute to high regional beta-diversity. Ecography, 41: 1256-1269. DOI: https://doi. org/10.1111/ecog.03126 
Eastman, J.R. 2012. IDRISI Selva: Guía para SIG y procesamiento de imágenes. Manual versión 17. Clark University, Worcester, MA. 321pp.

Foley, J.A.; Asner, G.P.; Costa, M.H.; Coe, M.T.; Defries, R.; Gibbs, H.K.; Howard, E.A.; Olson, S.; Patz, J.; Ramankutty, N.; Snyder, P. 2007. Amazonia revealed: forest degradation and loss of ecosystem goods and services in the Amazon Basin. Frontiers in Ecology and the Environment, 5(1): 25-32. DOI: https:// doi.org/10.1890/1540-9295(2007)5[25: ARFDAL]2.0.CO;2

Gallice, G.R.; Larrea-Gallegos, G.; Vázquez-Rowe, I. 2019. The threat of road expansion in the Peruvian Amazon. Oryx, 53(2): 284-292. DOI: https://doi.org/10.1017/S0030605317000 412

GTM-NDC. 2018. Grupo de Trabajo Multisectorial de naturaleza temporal encargado de generar información técnica para orientar la implementación de las Contribuciones Nacionalmente Determinadas (GTM-NDC): informe final. Ministerio del Ambiente, Lima, Perú. 929pp.

Goldstein, A.; Turner, W.R.; Spawn, S.A.; Anderson-

Teixeira, K.J.; Cook-Patton, S.; Fargione, J.; Gibbs, H.K.; Griscom, B.; Hewson, J.H.; Howard, J.F.; Ledezma, J.C.; Page, S.; Koh, L.P.; Rockström, J.; Sanderman, J.; Hole, D.G. 2020. Protecting irrecoverable carbon in Earth's ecosystems. Nature Climate Change, 10: 287-295. DOI: https://doi.org/10.1038/s41558-020-0738-8 Honorio Coronado, E.N.; Vega Arenas, J.E.; Corrales Medina, M.N. 2015. Diversidad, estructura y carbono de los bosques aluviales del noreste peruano. Folia Amazónica, 24(1): 55-70. DOI: https://doi.org/10.24841/fa.v2 4i1.59

Honorio Coronado, E.N.; Reyna, J.; Flores, G.; Grández, J.; Lähteenoja, O.; Draper, F.C.; Åkesson, C.M.; Baker, T.R.; Bhomia, R.K.; Cole, L.E.S.; Dávila, N.; del Águila, J.; del Águila, M.; del Castillo Torres, D.; Hastie, A.; Lawson, I.T.;
Martín Brañas, M.; Monteagudo, A.; Phillips, O.L.; Ramírez, E.; Ríos, M.; Ríos, S.; Rodriguez, L.; Roucoux, K.H.; Tagle Casapia, X.; Vasquez, R.; Montoya, M. (en revisión). Intensive field sampling increases the known extent of carbon-rich Amazonian peatland pole forests. Environmental Research Letters.

Hyman, G.; Barona, E. 2010. Roads and deforestation in the Central Peruvian Amazon. Poster CIAT, Colombia.

INEI. 2017. Centros poblados del censo 2017. Instituto Nacional de Estadística e Información. (http://sige.inei.gob.pe/test/atlas/). Acceso: 02/03/2020.

IPPC. 2006. 2006 IPCC Guidelines for National Greenhouse Gas Inventories. Prepared by the National Greenhouse Gas Inventories Programme. Eggleston H.S., Buendia L., Miwa K., Ngara T. and Tanabe K. (eds). IGES, Japan. $5 \mathrm{v}$.

Lähteenoja, O.; Ruokolainen, K.; Schulman, L.; Oinonen, M. 2009. Amazonian peatlands: an ignored C sink and potential source. Global Change Biology, 15: 2311-2320. DOI: https:// doi.org/10.1111/j.1365-2486.2009.01920.x

Lähteenoja, O.; Page, S. 2011. High diversity of tropical peatland ecosystem types in the Pastaza-Marañón basin, Peruvian Amazonia. Journal of Geophysical Research: Biogeosciences, 116: G02025. https://doi.org/ 10.1029/2010JG001508

Lähteenoja, O.; Reátegui, Y.R.; Räsänen, M.; Del Castillo Torres, D.; Oinonen, M.; Page, S. 2012. The large Amazonian peatland carbon sink in the subsiding Pastaza-Marañón foreland basin. Global Change Biology, 18: 164-178. https:// doi.org/10.1111/j.1365-2486.2011.02504.x

Laurance, W.F.; Goosem, M.; Laurance, S. 2009. Impacts of roads and linear clearings on tropical forests. Trends in Ecology \& Evolution, 24(12): 659-669. DOI: https://doi. org/10.1016/j.tree.2009.06.009 
Larrea-Gallegos, G.; Vázquez-Rowe, I.; Gallice, G. 2017. Life cycle assessment of the construction of an unpaved road in an undisturbed tropical rainforest area in the vicinity of Manu National Park, Peru. The International Journal of Life Cycle Assessment, 22, 1109-1124. DOI: https:// doi.org/10.1007/s11367-016-1221-7

Mäki, S.; Kalliola, R.; Vuorinen, K. 2001. Road construction in the Peruvian Amazon: process, causes and consequences. Environmental Conservation, 28(3): 199-214. DOI: https:// doi.org/10.1017/S0376892901000212

Malhi, Y.; Aragao, L.E.O.; Metcalfe, D.B.; Paiva, R.; Quesada, C.A.; Almeida, S.; Anderson, L.; Brando, P.; Chambers, J.Q.; da Costa, A.C.L.; Hutyra, L.R.; Oliveira, P.; Patiño, S.; Pyle, E.H.; Robertson, A.L.; Teixeira, L.M. 2009. Comprehensive assessment of carbon productivity, allocation and storage in three Amazonian forests. Global Change Biology, 15(5): 1255-1274. DOI: https://doi. org/10.1111/j.1365-2486.2008.01780.x

Martín Brañas, M.; Núñez Pérez, C.; Fabiano, E.; Del Aguila Villacorta, M.; Schulz, C.; Laurie, N.; Sanjurjo, J.; Davies, A.; Roucoux, K.; Lawson, I.; Andueza, L. 2019. Urarina: Identidad y memoria en la cuenca del río Chambira. Instituto de Investigaciones de la Amazonía Peruana, Iquitos, Perú. 75pp.

MINEDU. 2019. Red de ríos navegables de Perú. Ministerio de Educación. (https:// sigmed.minedu.gob.pe/descargas/). Acceso: 02/03/2020.

MINAM. 2019a. Mapa de la cobertura y pérdida de bosque húmedo amazónico al 2018. Programa Nacional de Conservación de Bosques para la Mitigación del Cambio Climático - Ministerio del Ambiente. (http://geobosques.minam. gob.pe). Acceso: 02/03/2020.

MINAM. 2019b. Inventario nacional de gases de efecto invernadero del año 2014 y actualización de las estimaciones de los años 2000, 2005, 2010 y 2012. Dirección General de Cambio Climático y Desertificación - Ministerio del Ambiente. Perú. 336pp.

MINAM. 2019c. Mapa de ecosistemas del Perú. Dirección General de Ordenamiento Territorial Ambiental - Ministerio del Ambiente. (https:// www.gob.pe/institucion/minam/informespublicaciones/277146-mapa-nacional-deecosistemas-del-peru). Acceso: 02/03/2020.

MTC. 2018. Red Vial Departamental 2018. Ministerio de Transportes y Comunicaciones. (https://portal.mtc.gob.pe/estadisticas/ descarga.html). Acceso: 02/03/2020.

NASA. 2000. Shuttle Radar Topography Mission digital topographic data. NASA, the National Geospatial-Intelligence Agency, and the German and Italian Space Agencies. (https://www2.jpl.nasa.gov/srtm/). Acceso: 02/03/2020.

Ochieng, R.M.; Arts, B.; Brockhaus, M.; VisserenHamakers, I.J. 2018. Institutionalization of REDD+ MRV in Indonesia, Peru, and Tanzania. Ecology and Society, 23(2): 8. DOI: https://doi. org/10.5751/ES-09967-230208

Oliveira, P.J.; Asner, G.P.; Knapp, D.E.; Almeyda, A.; Galván-Gildemeister, R.; Keene, S.; Raybin, R.F.; Smith, R.C. 2007. Land-use allocation protects the Peruvian Amazon. Science, 317(5842): 1233-1236. DOI: https://doi.org/10.1126/ science.1146324

Petroperu. 2019. Lotes petroleros en el Perú. (https://www.perupetro.maps.arcgis.com/ apps/webappviewer/index.html?id=6a830a 470b934f0687c8ed84c2bacacc). Acceso: 02/03/2020.

Provias Descentralizado. 2019. Topografía Alineamiento - Alternativa 1. Planta y Perfil - Prog. Km 0+00 al km 100+000 Carretera Nor Oriental Iquitos-Saramiriza [Plano]. 1:30,000. Lima. Ministerio de Transportes y Comunicaciones. 
Roucoux, K.H.; Lawson, I.T.; Baker, T.R.; Del Castillo Torres, D.; Draper, F.C.; Lähteenoja, O.; Gilmore, M.P.; Honorio Coronado, E.N.; Kelly, T.J.; Mitchard, E.T.A.; Vriesendorp, C.F. 2017. Threats to intact tropical peatlands and opportunities for their conservation. Conservation Biology, 31(6): 1283-1292. DOI: https://doi.org/10.1111/cobi.12925

Salo, M.; Pyhälä, A. 2007. Exploring the gap between conservation science and protected area establishment in the Allpahuayo-Mishana National Reserve (Peruvian Amazonia). Environmental Conservation, 34(1): 23-32. DOI: https://doi.org/10.1017/S037689290 7003700

Sánchez-Cuervo, A.M.; De Lima, L.S.; Dallmeier, F.; Garate, P.; Bravo, A.; Vanthomme, H. 2020. Twenty years of land cover change in the southeastern Peruvian Amazon: implications for biodiversity conservation. Regional Environmental Change, 20(1): 8. DOI: https:// doi.org/10.1007/s10113-020-01603-y

Schulz, C.; Matrtín Brañas, M.; Núñez Pérez, C.; Del Aguila Villacorta, M. D.; Laurie, N.; Lawson, I.T.; Roucoux, K.H. 2019. Uses, cultural significance, and management of peatlands in the Peruvian Amazon: Implications for conservation. Biological Conservation, 235: 189-198. DOI: https://doi.org/10.1016/j. biocon.2019.04.005

SERFOR. 2015. Bosques de Producción permanente. Servicio Forestal y de Fauna Silvestre. (https://www.geo.serfor.gob.pe/visor). Acceso: 02/03/2020.

SERNANP. 2019. Áreas Naturales Protegidas. Servicio Nacional de Áreas Naturales Protegidas. (https://www.geo.sernanp.gob. pe/visorsernanp/). Acceso: 02/03/2020.

Sullivan, M.J.; Talbot, J.; Lewis, S.L.; Phillips, O.L.; Qie, L.; Begne, S.K.; Chave, J.; Cuni-Sanchez, A.; Hubau, W.; Lopez-Gonzalez, G.; [...]; Zemagho, L. 2017. Diversity and carbon storage across the tropical forest biome. Scientific Reports, 7(1): 1-12. DOI: https://doi.org/10.1038/ srep39102

Vázquez-Rowe, I.; Kahhat, R.; Larrea-Gallegos, G.; Ziegler-Rodriguez, K. 2019. Peru's road to climate action: Are we on the right path? The role of life cycle methods to improve Peruvian national contributions. Science of The Total Environment, 659: 249-266. DOI: https://doi. org/10.1016/j.scitotenv.2018.12.322

Vicuña Miñano, E.; Baker, T. R.; Banda, K.; Honorio Coronado, E.; Monteagudo, A.; Phillips, O. L.; Del Castillo Torres, D.; Farfan Rios, W.; Flores, G.; Huaman, D.; Tantte Huaman, K.; Hidalgo Pizango, G.; Lojas Aleman, E.; Melo, J.B.; Pickavance, G.C.; Rios, M.; Rojas, M.; Salinas, N.; Vasquez Martinez, R. 2018. El sumidero de carbono en los bosques primarios amazónicos es una oportunidad para lograr la sostenibilidad de su conservación. Folia Amazónica, 27(1): 101-109. DOI: https://doi. org/10.24841/fa.v27i1.456

Vijay, V.; Reid, C.D.; Finer, M.; Jenkins, C.N.; Pimm, S.L. 2018. Deforestation risks posed by oil palm expansion in the Peruvian Amazon. Environmental Research Letters, 13(11): 114010. DOI: https://doi.org/10.1088/17489326/aae540.

Vilela, T.; Harb, A.M.; Bruner, A.; Da Silva Arruda, V.L.; Ribeiro, V.; Alencar, A.A.C.; Escobedo Grandez, A.J.; Rojas, A.; Laina, A.; Botero, R. 2020. A better Amazon road network for people and the environment. Proceedings of the National Academy of Sciences, 117(13): 7095-7102. DOI: https://doi.org/10.1073/ pnas. 1910853117

Vittor, A.Y.; Gilman, R.H.; Tielsch, J.; Glass, G.; Shields, T.I.M.; Lozano, W.S.; Pinedo-Cancino, V.; Patz, J.A. 2006. The effect of deforestation on the human-biting rate of Anopheles darlingi, the primary vector of falciparum malaria in the Peruvian Amazon. The American 
Journal of Tropical Medicine and Hygiene, 74(1): 3-11. DOI: https://doi.org/10.4269/ ajtmh.2006.74.3

Warren, M.; Hergoualc'h, K.; Kauffman, J.B.; Murdiyarso, D.; Kolka, R. 2017. An appraisal of Indonesia's immense peat carbon stock using national peatland maps: uncertainties and potential losses from conversion. Carbon Balance and Management, 12(1): 12. DOI: https://doi.org/10.1186/s13021-017-0080-2 Watson, J.E.; Evans, T.; Venter, O.; Williams, B.; Tulloch, A.; Stewart, C.; Thompson, I.; Ray, J.C.;
Murray, K.; Salazar, A.; Mcalpine, C.; Potapov, P.; Walston, J.; Robinson, J.G.; Painter, M.; Wilkie, D.; Filardi, C.; Laurance, W.F.; Houghton, R.A.; Maxwell, S.; Grantham, H.; Samper, C.; Wang, S.; Laestadius, L.; Runting, R.K.; Silva-Chávez, G.A.; Ervin, J.; Lindenmayer, D. 2018. The exceptional value of intact forest ecosystems. Nature Ecology \& Evolution, 2(4): 599-610. DOI: https://doi.org/10.1038/s41559-0180490-x

Recibido: 30 de julio de 2020 Aceptado para publicación: 18 de setiembre de 2020

Esta obra está bajo una Licencia Creative Commons Atribución-NoComercial-SinDerivar 4.0 Internacional. 